Case Report

\title{
Acquired Immunodeficiency Syndrome Presented as Atypical Ocular Toxoplasmosis
}

\author{
Elias Khalili Pour $\left(\mathbb{D},{ }^{1}\right.$ Hamid Riazi-Esfahani $\mathbb{D}^{1}{ }^{1}$ Nazanin Ebrahimiadib ${ }^{\mathbb{D}}{ }^{1}$ \\ Violet Zaker Esteghamati $\oplus^{2},{ }^{2}$ and Mohammad Zarei $\oplus^{1}$ \\ ${ }^{1}$ Eye Research Center, Farabi Eye Hospital, Tehran University of Medical Sciences, Tehran, Iran \\ ${ }^{2}$ Imam Khomeini General Hospital, School of Medicine, Tehran University of Medical Sciences, Tehran, Iran \\ Correspondence should be addressed to Mohammad Zarei; mzareiran81@gmail.com
}

Received 28 January 2021; Revised 16 April 2021; Accepted 24 April 2021; Published 3 May 2021

Academic Editor: Kamal Kishore

Copyright (c) 2021 Elias Khalili Pour et al. This is an open access article distributed under the Creative Commons Attribution License, which permits unrestricted use, distribution, and reproduction in any medium, provided the original work is properly cited.

\begin{abstract}
Background. The aim is to report an atypical presentation of ocular toxoplasmosis which led to the diagnosis of Acquired Immunodeficiency Syndrome (AIDS). Case Report. The 38-year-old woman was referred with metamorphopsia and reduced vision in the right eye over the past 3 weeks. Slit-lamp examination revealed granulomatous keratic precipitates (KPs), 2+ cells in the anterior chamber, and posterior synechiae. Fundus examination was remarkable for a white patch surrounding a scar, inferonasal to the optic disc with fibrous bands emanating from the lesion, and the retina around this region was detached with considerable extension towards the periphery, while no breaks could be appreciated. She mentioned anorexia and losing $10 \mathrm{~kg}$ in the past three months, and signs of anemia like paleness of face skin, bed nails, and bilateral angular cheilitis were observed on systemic evaluation. The results of the patient's complete blood count revealed anemia and leukopenia and CD4 lymphocytes: 79 cells $/ \mu$ L. Enzyme-linked immunosorbent assays (ELISA) for HIV antibodies came back positive which was later confirmed with the Western blot test. Brain magnetic resonance imaging (MRI) showed multiple ring-enhancing lesions in both cerebral cortices. The patient underwent antitoxoplasmosis and anti-HIV treatment and serous retinal detachment completely resolved. Conclusion. This report highlights the fact that sometimes, the eyes are the site of the first presentation of a systemic lifethreatening condition and emphasizes the role of ophthalmologists in such cases. In cases of atypical presentation, appropriate laboratory tests and CNS imaging should be requested. Systemic treatment with antitoxoplasmosis regimens and highly active antiretroviral therapy (HAART) is mandatory in AIDS patients with ocular toxoplasmosis.
\end{abstract}

\section{Background}

Since the 1950s, ocular toxoplasmosis has been reported as the most common cause of infectious uveitis worldwide. Toxoplasma gondii is a ubiquitous obligate intracellular microorganism, which is estimated to affect one-third of the world's population [1-3].

Active ocular toxoplasmosis is characterized by a necrotizing retinochoroiditis lesions accompanied by localized or diffuse vitritis. In immunocompetent patients, lesions are usually self-limited and resolve within two months. Atypical forms of ocular toxoplasmosis such as diffuse outer retinitis, which can mimic acute retinal necrosis (ARN), punctuate outer retinal toxoplasmosis, occlusive retinal vasculitis, neu- roretinitis, scleritis, and exudative retinal detachment, have been reported [4]. Atypical lesions may occur bilaterally and have been reported in the immunocompromised, neonates, and elderly patients [5].

This parasite can be fatal in immunocompromised individuals, such as HIV/AIDS patients especially those with CD4 T lymphocyte cell counts less than 200 cells $/ \mu \mathrm{L}$. In such a setting, reactivation of opportunistic organisms like Toxoplasma gondii can occur with multiorgan involvement, especially central nervous system (CNS), and with much lower incidence, ocular involvement [5-7].

The central nervous system, as the most common site of Toxoplasma reactivation, can be involved in about $30 \%$ of patients with AIDS, especially in those who are 


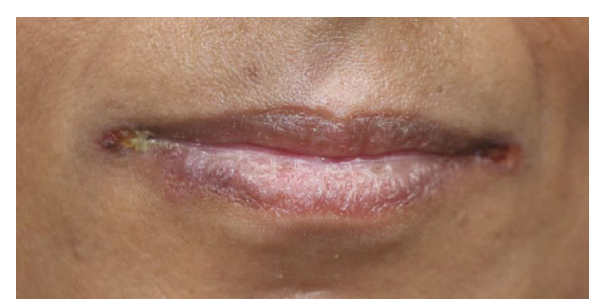

(a)

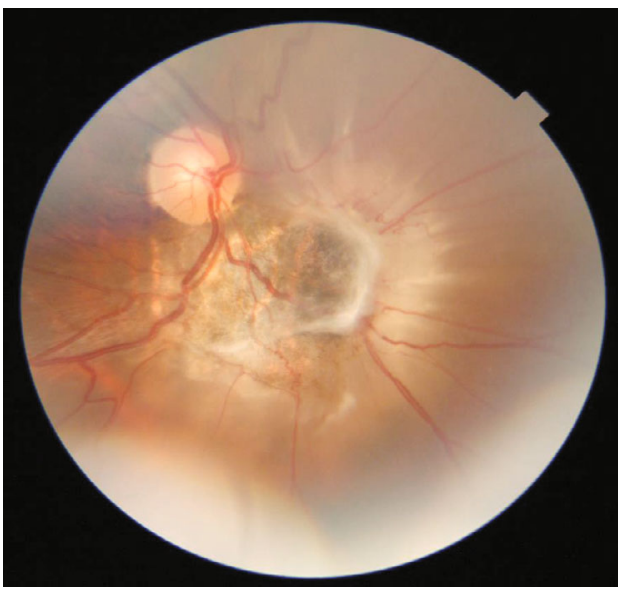

(c)

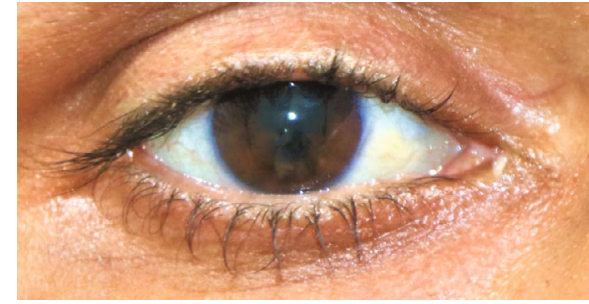

(b)
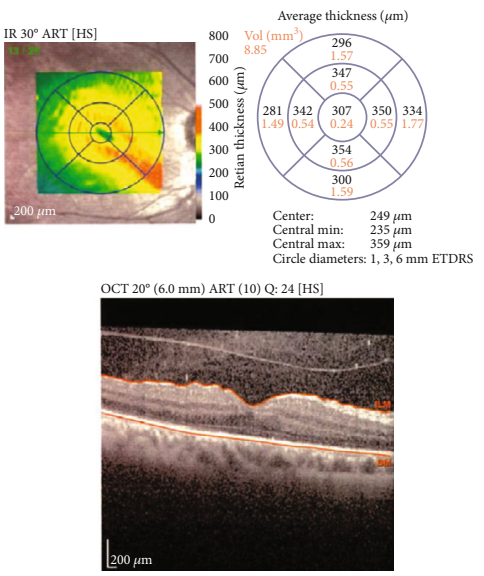

(d)

FIGURE 1: Clinical features of the patient at the time of presentation. Bilateral angular cheilitis (a), posterior synechiae, and keyhole-shaped pupil (b), In the right eye inferonasal to the optic disc, an annular white patch centered with a pigmented scar is seen. White subretinal bands are extending from the lesion. The size of the lesion is about three-disc diameter, and the adjacent retina is detached (c). Macular optical coherence tomography of the right eye demonstrates evidence of vitritis, detached posterior hyaloid, and a fine epiretinal membrane nasal to the fovea (d).

treatment-naive and Toxoplasma seropositive with CD4 $<100$ cells/ $\mu \mathrm{L}[8-11]$.

Here, we report an atypical presentation of ocular toxoplasmosis with a serous retinal detachment which led to the diagnosis of AIDS. The serous retinal detachment resolved with antitoxoplasmosis treatments and HAART.

\section{Case Report}

The 38-year-old woman was referred to the retina department of Farabi Eye Hospital with metamorphopsia and reduced vision in the right eye over the past 3 weeks. At the time of the presentation, she mentioned anorexia and losing $10 \mathrm{~kg}$ in the past three months, and signs of anemia like paleness of face skin, bed nails, and bilateral angular cheilitis were observed (Figure 1(a)). Uncorrected visual acuity in the right eye was counting fingers at two meters. Posterior synechia (PS) and pigment deposit over the anterior crystalline lens capsule precluded precise refraction in this eye. Slit-lamp examination revealed granulomatous keratic precipitates (KPs) distributed in Arlt's triangle, $2+$ cells in the anterior chamber, and a relatively broad-based PS causing a keyhole appearance in the pupil (Figure 1(b)). The crystalline lens was clear and $2+$ cells were present in the anterior vitreous. Fundus examination was remarkable for a white patch surrounding a scar, inferonasal to the optic disc with threedisc diameter size. Some fibrous bands were emanating from the lesion, and the retina around this region was detached with considerable extension towards the superior, nasal, and inferior periphery, while no breaks could be appreciated (Figure 1(c)). The vision in the left eye was 20/20, and ophthalmic examination was unremarkable in this eye.

Spectral-domain optical coherence tomography (SDOCT) (Spectralis Heidelburg Germany) disclosed evidence of vitritis, a detached posterior hyaloid face, and a fine epiretinal membrane (ERM) nasal to the fovea in the right eye (Figure 1(d)).

She denied using any medications or having other illnesses. Considering systemic symptoms and signs and due to involuntary weight loss, the patient underwent a comprehensive infectious, neoplastic, and rheumatologic workup. The results of the patient's complete blood count (CBC) are summarized in Table 1. Based on the ocular findings, additional tests were ordered for Toxoplasma gondii antibodies which revealed the presence of IgG antibodies $(18 \mathrm{IU} / \mathrm{mL}$; reference range: positive $\geq 12 \mathrm{IU} / \mathrm{mL}$, suspicious $10-11$ $9 \mathrm{IU} / \mathrm{mL}$, negative $\leq 9 \mathrm{IU} / \mathrm{mL}$ ) while IgMs were absent $(0.1 \mathrm{IU} / \mathrm{mL}$; reference range: positive $\geq 0.65 \mathrm{IU} / \mathrm{mL}$, suspicious $0.55-0.64 \mathrm{IU} / \mathrm{mL}$, negative $\leq 0.55 \mathrm{IU} / \mathrm{mL}$ ).

Imaging studies including chest-X-ray, abdominal sonography, and age-related neoplastic workup including mammography, Pap smear, and colonoscopy were unremarkable except for nonspecific polyps in the patient's large intestine. 
TABLE 1: The results of the patient's CBC-diff.

\begin{tabular}{lcc}
\hline Blood component & & Reference range \\
\hline White blood cells & 3400 & $4500-11,000 / \mathrm{mm}^{3}$ \\
Red blood cells & 4.19 & Male: $4.3-5.9 \mathrm{million}^{3} \mathrm{~mm}^{3}$ \\
& & Female: $3.5-5.5 \mathrm{million} / \mathrm{mm}^{3}$ \\
Hemoglobin & 8.1 & Male: $13.5-17.5 \mathrm{~g} / \mathrm{dL}$ \\
& & Female: $12.0-16.0 \mathrm{~g} / \mathrm{dL}$ \\
Hematocrit & 26.8 & Male: $41 \%-53 \%$ \\
Mean corpuscular volume & 64.0 & Female: $36 \%-46 \%$ \\
Mean corpuscular hemoglobin & 19.3 & $80-100 \mu \mathrm{m}^{3}$ \\
Mean corpuscular hemoglobin concentration & 30.2 & $25.4-34.6 \mathrm{pg} / \mathrm{cell}$ \\
Platelets & 236000 & $31 \%-36 \% \mathrm{Hb} / \mathrm{cell}$ \\
Neutrophil & 61.7 & $150,000-400,000 / \mathrm{mm}{ }^{3}$ \\
Lymphocytes & 27.9 & $40-60 \%$ \\
Monocytes & 7.8 & $20-40 \%$ \\
Eosinophil & 2.6 & $4-8 \%$ \\
Basophil & 0 & $1-3 \%$ \\
CD4 lymphocytes & 79 cells $/ \mu \mathrm{L}$ & $0-1 \%$ \\
\hline
\end{tabular}

Enzyme-linked immunosorbent assays (ELISA) for HIV antibodies came back positive which was later confirmed with the Western blot test.

Once the diagnosis of toxoplasmic retinochoroiditis in an immunosuppressed patient was established, treatment with trimethoprim/sulfamethoxazole (TMP/SMX) 160/800 mg twice daily was commenced. Brain magnetic resonance imaging (MRI) showed multiple ring-enhancing lesions in both cerebral cortices (Figure 2). Meanwhile, three weeks after initial ophthalmic presentations, the patient developed a right hemiparesis. Due to the numerous lesions in the patient's motor cortex in the left and right lobes, the diagnosis of toxoplasmic encephalitis was made for the patient.

Highly active antiretroviral therapy (HAART) was added to her antitoxoplasmosis treatment four weeks after initial presentation and the dose of trimethoprim/sulfamethoxazole (TMP/SMX) increased to $160 / 800 \mathrm{mg} /$ three times a day and azithromycin $250 \mathrm{mg} /$ daily was added to the antitoxoplasmic regimen. The systemic steroid was not added to the patient's treatment regimen due to concerns about the patient's immunosuppression. Two months after initial presentation, vitreous inflammation decreased substantially, subretinal fluid gradually resolved, and the uncorrected visual acuity improved to 20/100 (Figure 3). Yellow subretinal hard exudates appeared in the perifoveal area with complete reattachment of the retina in three months. Two months after starting HAART, the patient's hemiparesis improved significantly during this time.

\section{Discussion}

The presence of cells in the anterior chamber and vitreous cavity, large Kps, PS, and a patch of retinitis adjacent to a pigmented retinochoroidal scar are clinical clues to the diagnosis of toxoplasmic retinochoroiditis. According to the fundus examination, weight loss, anemia, and leukopenia of the patient, internal medicine and infectious disease service consultation were requested. The patient's neuroimaging and the appearance of typical ring-enhanced lesions besides positive HIV test and diminished CD4+ cells suggested a possible diagnosis of toxoplasmosis in the patient, and therefore, no intraocular fluid PCR test requested for the patient. Positive Toxoplasma IgG antibody along with negative IgM indicates infection with the organism at some time $[2,12,13]$. According to a meta-analysis conducted by Borna et al., for estimating the level of immunity to toxoplasmosis in reproductive ages in Iran, the overall estimation for the prevalence of antitoxoplasma total antibody was 39.9\% (95\% CI: 26.1-53.7) [14].

However, this case shows some ocular and systemic features that are not commonly expected in the typical case of toxoplasmic retinochoroiditis. In this case, pursuing these atypical features led us to the diagnosis of AIDS.

The first red flag was the presence of an extensive retinal detachment with tractional bands surrounding the central scar and no responsible break which could be suggestive of tractional and/or exudative retinal detachment in our case. Although tractional, exudative, and rhegmatogenous retinal detachments can occur in ocular toxoplasmosis, such reports are limited; the reported prevalence of retinal detachment in acquired toxoplasmosis varies between 2.5 and 11 percent which is mostly of tractional or rhegmatogenous type [13, 15-17].

There are several reports on the management of retinal detachment in the setting of ocular toxoplasmosis. Among the 193 patients with ocular toxoplasmosis, Kianersi et al. reported five patients with $\mathrm{RD}$ : three rhegmatogenous and two tractional. All five patients underwent vitrectomy or scleral buckling surgery. It is well known that severe intraocular inflammation can lead to tractional retinal detachment. With the progression of traction, a retinal hole and subsequent rhegmatogenous detachment may develop. Another possible mechanism of hole formation is the occurrence of full-thickness necrosis in the retinitis patch [17]. 


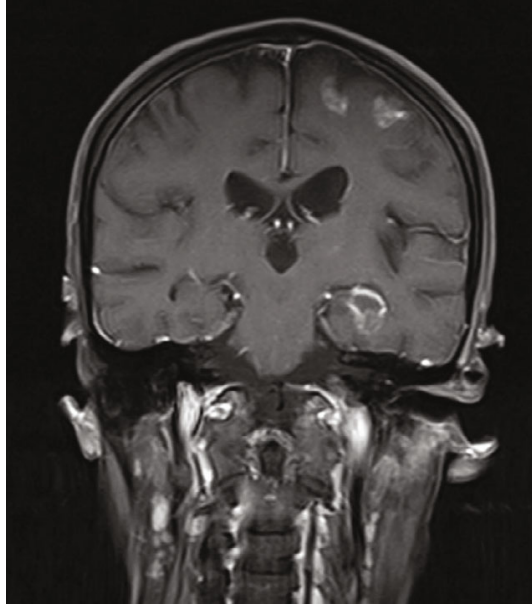

(a)

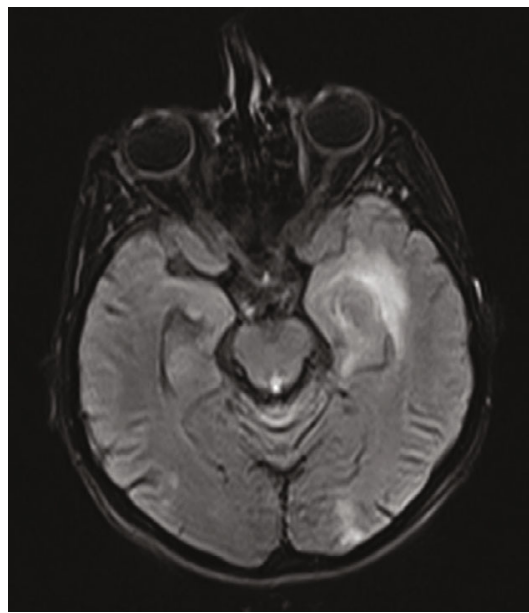

(b)

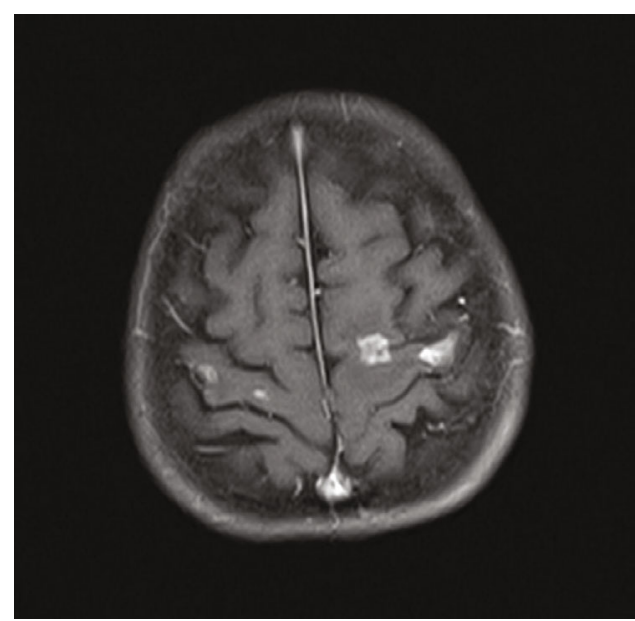

(c)

FIGURE 2: Magnetic resonance imaging (MRI): T1 with contrast in coronal (a) and axial planes (b, c): multiple ring-enhancing lesions in the left and right cerebral cortices.

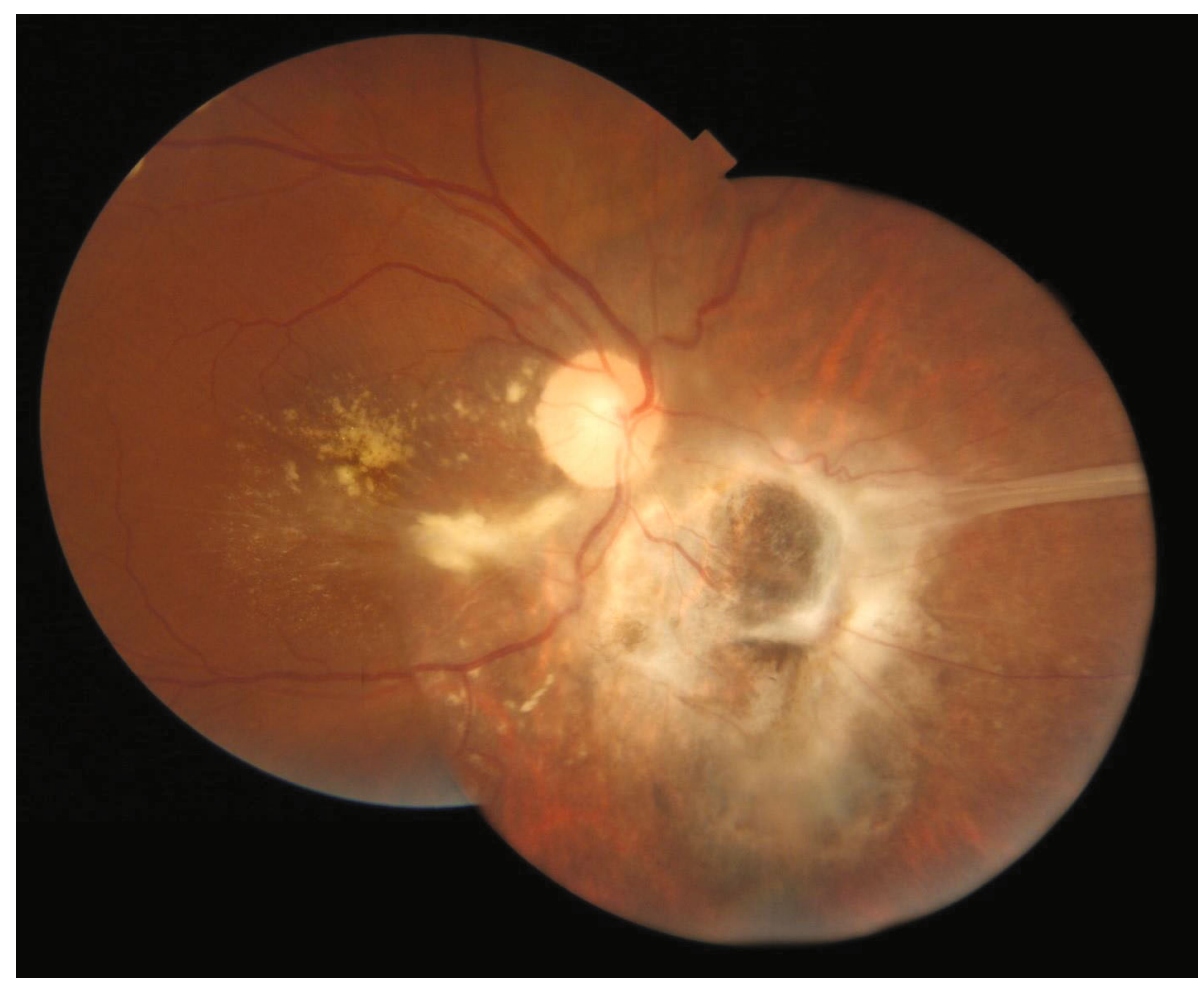

FIGURE 3: Montage fundus photography of the patient's right eye three months after initiation of antitoxoplasmosis treatment and HAART shows complete retinal reattachment. A retinal fold extends from the lesion to the nasal periphery. Subretinal exudates are seen in the inferior area of the disc and around the macula.

In another study, $\mathrm{Al}$-Zahrani et al. reported a 24-year-old man with exudative RD secondary to reactivation of a toxoplasmosis lesion, which was successfully managed with antitoxoplasmosis treatment [15].

In a study by Faridi et al., on thirty-five eyes of 28 patients with ocular toxoplasmosis and sufficient follow-up, they identified 4 eyes (11 percent) with secondary RD, which was either rhegmatogenous, tractional, or a combination of the two. All eyes with retinal detachment underwent surgical repair of which $50 \%$ developed recurrent RDs (two of 4 patients). They concluded that handling ocular toxoplasmosis and related RDs may necessitate several measures, including surgical management, systemic therapy, and intravitreal injections. Formation of new breaks or PVR-related complications may necessitate multiple surgeries in cases with recurrent $\mathrm{RD}$ [16]. Our case represents the exudative detachment with no distinct retinal break. Observation of a retinal fold may advocate the presence of a tractional component 
in this case. The resolution of $\mathrm{RD}$ following an antitoxoplasmic regimen and HAART and without surgical intervention may suggest that decision upon surgery should not be rushed in such patients, and a period of close follow-up visits for ocular examination combined with appropriate systemic treatment may be warranted.

Angular cheilitis and significant weight loss are not expected to occur in typical ocular toxoplasmosis. The presence of angular cheilitis calls for investigating the possibility of anemia and its underlying etiology. In our case, CBC revealed hypochromic microcytic anemia suggestive of iron-deficiency anemia and leukopenia which necessitates a thorough investigation for immunodeficiency.

Increased energy expenditure happens in the setting of opportunistic infections. Weight loss in patients with HIV is associated with poor prognosis, increased risk of disease progression, and opportunistic pathology [18].

In a study by Neves et al. on 37 immunocompetent patients with acute acquired toxoplasmosis, the frequency of systemic manifestations was inspected. Weight loss, anemia, and leukopenia were seen in $62.2 \%, 10.8 \%$, and $16.2 \%$ of patients, respectively [12]. Although our patient was not a case of acquired toxoplasmosis and the results of the study by Neves et al. could not be generalized to reactivated congenital toxoplasmosis, another similar study could investigate the possibility of anemia, leukopenia, and weight loss in this group of patients.

The possibility of toxoplasmic encephalitis should be investigated in every patient with HIV and retinochoroidal toxoplasmosis $[19,20]$. The study of choice for this purpose is brain MRI with contrast that can illustrate characteristic enhancing ring lesions. While not pathognomonic, the presence of several brain abscesses is the most characteristic feature of T. gondii infection in AIDS patients. Autopsy usually shows a global involvement of both hemispheres, though the basal ganglia and the corticomedullary junction are the most common sites of involvement [9-11].

This report highlights the fact that sometimes the eyes are the site of the first presentation of a systemic life-threatening condition and emphasizes the role of ophthalmologists in such cases. Judicious ocular and systemic evaluation of patients with ocular toxoplasmosis are of utmost importance. In cases of atypical presentation, appropriate laboratory tests and CNS imaging should be requested. Systemic treatment with antitoxoplasmosis regimens and HAART is mandatory in AIDS patients with ocular toxoplasmosis. Treatment options for retinal detachment in this setting should be meticulously approached.

\section{Data Availability}

The data generated during the present study is available upon request from the corresponding author.

\section{Conflicts of Interest}

The authors declare that they have no conflicts of interest.

\section{References}

[1] S. Abgrall, C. Rabaud, and D. Costagliola, "Incidence and risk factors for toxoplasmic encephalitis in human immunodeficiency virus-infected patients before and during the highly active antiretroviral therapy era," Clinical Infectious Diseases, vol. 33, no. 10, pp. 1747-1755, 2001.

[2] G. N. Holland, "Ocular toxoplasmosis: a global reassessment. Part I: epidemiology and course of disease," American Journal of Ophthalmology, vol. 136, no. 6, pp. 973-988, 2003.

[3] C. S. Subauste, D. Ajzenberg, and A. Kijlstra, "Review of the series "Disease of the year 2011: toxoplasmosis" pathophysiology of toxoplasmosis," Ocular Immunology and Inflammation, vol. 19, no. 5, pp. 297-306, 2011.

[4] J. R. Smith and E. T. J. Cunningham, "Atypical presentations of ocular toxoplasmosis," Current Opinion in Ophthalmology, vol. 13, no. 6, pp. 387-392, 2002.

[5] Y.-H. Park and H.-W. Nam, "Clinical features and treatment of ocular toxoplasmosis," The Korean Journal of Parasitology, vol. 51, no. 4, pp. 393-399, 2013.

[6] M.-H. Heinemann, J. M. W. Gold, and J. Maisel, "Bilateral toxoplasma retinochoroiditis in a patient with acquired immune deficiency syndrome," Retina, vol. 6, no. 4, pp. 224227, 1986.

[7] K. F. Tabbara, "Ocular toxoplasmosis," International Ophthalmology, vol. 14, no. 5-6, pp. 349-351, 1990.

[8] F. J. San-Andres, R. Rubio, J. Castilla et al., "Incidence of acquired immunodeficiency syndrome-associated opportunistic diseases and the effect of treatment on a cohort of 1115 patients infected with human immunodeficiency virus, 19891997," Clinical Infectious Diseases, vol. 36, no. 9, pp. 11771185, 2003.

[9] C. Renold, A. Sugar, J. P. Chave et al., "Toxoplasma encephalitis in patients with the acquired immunodeficiency syndrome," Medicine (Baltimore), vol. 71, no. 4, pp. 224-239, 1992.

[10] S. B. Porter and M. A. Sande, "Toxoplasmosis of the central nervous system in the acquired immunodeficiency syndrome," The New England Journal of Medicine, vol. 327, no. 23, pp. 1643-1648, 1992.

[11] B. J. Luft and J. S. Remington, "Toxoplasmic encephalitis in AIDS," Clinical Infectious Diseases, vol. 15, no. 2, pp. 211222, 1992.

[12] E. S. Neves, L. N. Bicudo, A. L. Curi et al., "Acute acquired toxoplasmosis: clinical-laboratorial aspects and ophthalmologic evaluation in a cohort of immunocompetent patients," Memórias do Instituto Oswaldo Cruz, vol. 104, no. 2, pp. 393-396, 2009.

[13] L. E. H. Bosch-Driessen, T. T. J. M. Berendschot, J. V. Ongkosuwito, and A. Rothova, "Ocular toxoplasmosis: clinical features and prognosis of 154 patients," Ophthalmology, vol. 109, no. 5, pp. 869-878, 2002.

[14] S. Borna, M. Shariat, M. Fallahi, and L. Janani, "Prevalence of immunity to toxoplasmosis among Iranian childbearing age women: systematic review and meta-analysis," Iranian Journal of Reproductive Medicine, vol. 11, no. 11, pp. 861-868, 2013.

[15] Y. A. Al-Zahrani, H. A. Al-Dhibi, and A. A. Al-Abdullah, "Atypical presentation of ocular toxoplasmosis: a case report of exudative retinal detachment and choroidal ischemia," Middle East African Journal of Ophthalmology, vol. 23, no. 1, pp. 150-152, 2016. 
[16] A. Faridi, S. Yeh, E. B. Suhler, J. R. Smith, and C. J. Flaxel, "Retinal detachment associated with ocular toxoplasmosis," Retina, vol. 35, no. 2, pp. 358-363, 2015.

[17] F. Kianersi, A. Naderi Beni, H. Ghanbari, and F. Fazel, "Ocular toxoplasmosis and retinal detachment: five case reports," European Review for Medical and Pharmacological Sciences, vol. 16, Suppl 4, pp. 84-89, 2012.

[18] V. K. Sashindran and R. Thakur, "Malnutrition in HIV/AIDS: aetiopathogenesis," in Nutrition and HIVIAIDS, N. Dumais, Ed., IntechOpen, Rijeka, 2020.

[19] T. J. Stokkermans and S. J. Havens, Toxoplasma Retinochoroiditis, StatPearls Publishing, 2021, http://www.ncbi.nlm.nih .gov/pubmed/29630234. Accessed April 15, 2021.

[20] S. B. Murugan, "Commentary: ocular toxoplasma: from bench-side to clinical practice!," Indian Journal of Ophthalmology, vol. 66, no. 10, pp. 1489-1490, 2018. 